\title{
INFLUENCE OF ROTATING MAGNETIC FIELD ON GAS-LIQUID VOLUMETRIC MASS TRANSFER COEFFICIENT
}

\author{
Rafał Rakoczy ${ }^{* 1}$, Maciej Konopacki ${ }^{1}$, Marian Kordas ${ }^{1}$, Radosław Drozd ${ }^{2}$, Karol \\ Fijałkowski $^{2}$
${ }^{1}$ West Pomeranian University of Technology, Szczecin, Faculty of Chemical Engineering, Institute Poland \\ of Chemical Engineering and Environmental Protection Processes, al. Piastów 42, 71-065 Szczecin, \\ ${ }^{2}$ West Pomeranian University of Technology, Szczecin, Faculty of Biotechnology and Animal \\ Husbandry, Department of Immunology, Microbiology and Physiological Chemistry, al. Piastów 45, \\ 70-311 Szczecin, Poland
}

\begin{abstract}
The main objective of these experiments was to study the oxygen mass transfer rate through the volumetric mass transfer coefficient $\left(k_{L} a\right)$ for an experimental set-up equipped with a rotating magnetic field (RMF) generator and various liquids. The experimental results indicated that $k_{L} a$ increased along the magnetic strength and the superficial gas velocity. Mathematical correlations defining the influence of the considered factors on $k_{L} a$ were proposed.
\end{abstract}

Keywords: mass transfer; volumetric mass transfer coefficient; rotating magnetic field

\section{INTRODUCTION}

In many biotechnological processes oxygen supplied to a liquid is insufficient to meet the demand of microorganisms. The oxygen transfer rate is often the limiting factor in bioprocesses due to the low solubility in the medium (Karimi et al., 2013). The oxygen supply into a liquid constitutes one of the decisive factors for microbial growth and can play an important role in optimization of aeration of microbial culture (Galaction et al., 2004a). It should be noticed that the gas-liquid mass transfer is generally the rate-limiting step in many industrial processes (Mills et al., 1999). Therefore, it is important to ensure an adequate delivery of oxygen to the culturing media.

The oxygen mass transfer, described and analyzed by using the volumetric mass transfer coefficient, $k_{L} a$, is controlled by oxygen solubility into liquids. This parameter may be affected by a lot of factors, such as geometrical and operational characterization of the mixing system. Generally, mechanically agitated gas-liquid contractors are frequently applied in chemical processes or biochemical industries. Mechanical agitation can promote the mass transfer rate because it increases interfacial area between the liquid and gas phases (Garcia-Ochoa et al., 2009; Torab-Mostaedi et al., 2008).

There have been various strategies to improve the oxygen transfer rate in mixers. The novel approach for a mixing process was based on the application of a rotating magnetic field (RMF). As reported by Rakoczy et al. (2011), the RMF can augment the process intensity instead of mechanical mixing. Magnetic fields can be applied to induce in vivo and in vitro effects in many biological systems (Rakoczy et al., 2016). Recently, increasing attention is directed towards bio-magnetic stimulation of microorganisms using various types of magnetic fields (El-Gaddar et al., 2013; Gorobets et al., 2013; 
Santos et al., 2010). Magnetically assisted bioprocesses are conducted using a non-conventional stimulation of classical bioprocesses by various types of magnetic field (Hristov et al., 2011). It should be noted that RMF can be a versatile option for enhancing molecular transport and diffusion in aqueous culture media (Hajiani et al., 2013b).

Weng et al. (2011) pointed out that the increase of magnetic strength enhanced the volumetric mass transfer coefficient $\left(k_{L} a\right)$. Al-Qodah et al. (2000) found that this parameter becomes higher as the gas velocity and the magnetic strength increase. Chen et al. (2001) presented that the $k_{L} a$ was enhanced by the magnetic strength. A recent study by Hajiani et al. (2013a) involved the influence of RMF on the gas-liquid transfer. These results demonstrated that the mass transfer rate was enhanced due to nanoconvective stirring using magnetic nanoparticles.

The main aim of this work was to study the influence of RMF on the aeration process of distilled water and Herstin-Schramm (HS) medium and to work out some mathematical correlation for oxygen transfer in the presence of the external magnetic field (MF). The proposed equations may be useful for optimization or scaling-up of aeration efficiency under the action of RMF.

\section{EXPERIMENTAL DETAILS}

A schematic of the experimental set-up used in the presented study is shown in Fig. 1.

A total of $4 \mathrm{dm}^{3}$ of the working liquid was introduced into a vessel (3). The distilled water and HerstinSchramm (HS) medium composed of glucose $(2 \mathrm{w} / \mathrm{v} \%)$, yeast extract $(0.5 \mathrm{w} / \mathrm{v} \%)$, bacto-pepton $(0.5 \mathrm{w} / \mathrm{v} \%)$, citric acid $\left(0.115 \mathrm{w} / \mathrm{v} \%\right.$, ) $\mathrm{Na}_{2} \mathrm{HPO}_{4}(0.27 \mathrm{w} / \mathrm{v} \%), \mathrm{MgSO}_{4} \cdot 7 \mathrm{H}_{2} \mathrm{O}(0.05 \mathrm{w} / \mathrm{v} \%)$ and ethanol $(1 \mathrm{v} / \mathrm{v} \%)$ added after sterilization of the base (Ciechańska et al., 1998) were used as experimental liquids. The HS medium is commonly used as the growth medium in the process production of bacterial cellulose (Fijałkowski et al., 2015). The liquid phase height and temperature was equal to $0.3 \mathrm{~mm}$ and $20^{\circ} \mathrm{C}$, respectively.

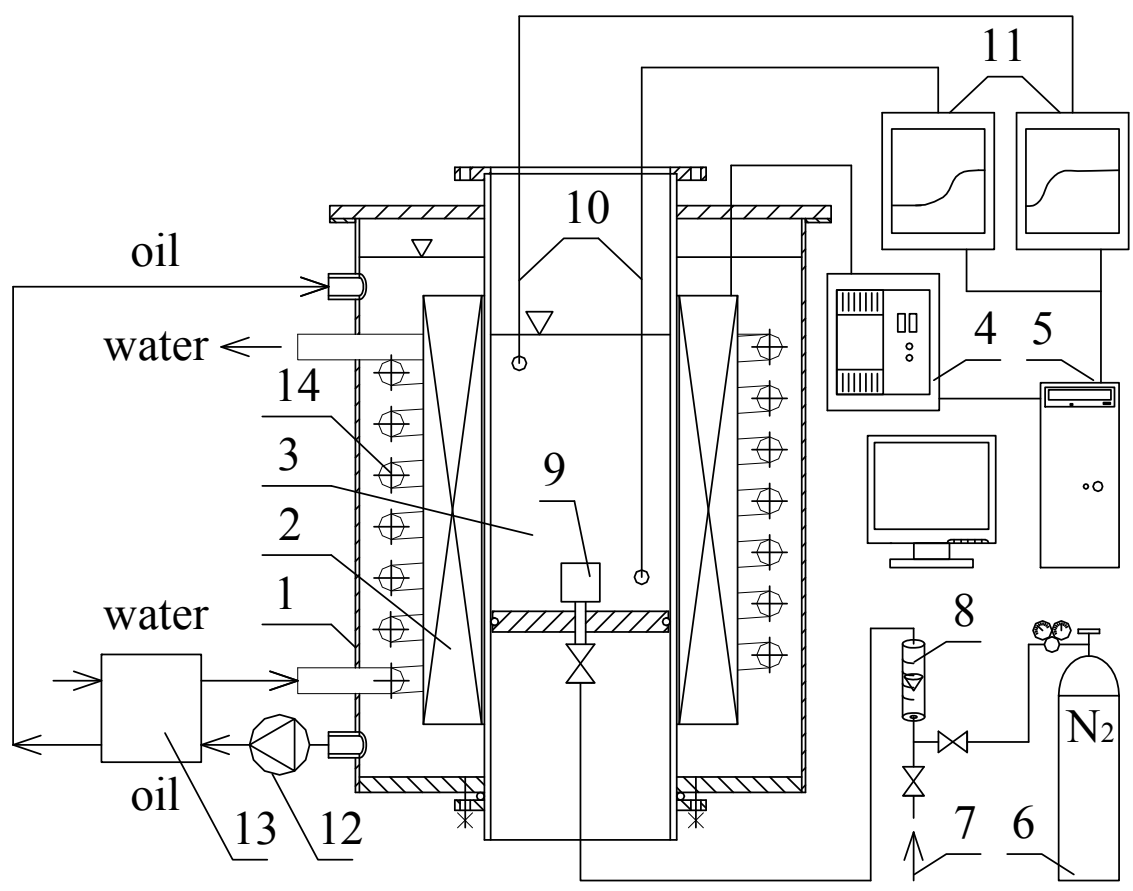

Fig. 1. Schematic diagram of the experimental set-up;

1 - housing, 2 - RMF generator, 3 - vessel, 4 - a.c. transistorized inverter, 5 - personal computer,

6 - nitrogen cylinder, 7 - air supply, 8 - rotameter, 9 - membrane sparger, 10 - oxygen probes, 11 - recorders, 12 - circulating pump, 13 - heat exchanger, 14 - internal coil 
Nitrogen gas (6) was used for oxygen elimination from the experimental set-up. A rotameter (8) was applied in order to monitor the flow of nitrogen and the average supply air flow rate (7). Gas was injected into the tested liquids by means of a membrane sparger (9). The membrane enabled to obtain fine bubbles with narrow size distribution (in the range between 0.5 and $3 \mathrm{~mm}$ ). The gas (air) flow rate was varied between 1 and $5 \mathrm{dm}^{3} \cdot \mathrm{min}^{-1}$. The superficial gas velocity was changed in the range between $0.001-0.005 \mathrm{~m} \cdot \mathrm{s}^{-1}$.

Oxygen probes (10) and recorders (11) were used to measure changes in dissolved oxygen concentration. The solved oxygen concentration in the working liquids was measured using CX-701 multifunction meters with COG-1 probes (Elmetron, Poland). It was assumed that the response of the oxygen electrode to the change in the oxygen concentration is sufficiently fast and does not affect the determination accuracy (Galaction et al., 2004b; Montes et al., 1999; Ozbek and Gayik, 2001).

The temperature of the working liquid during the exposition to RMF was controlled by an additional cooling system based on oil circulation (in a heat exchanger (13) a pump (12)) and water circulation in an internal coil (14).

The active power for the RMF system was calculated by the following relation (Rakoczy, 2013):

$$
P=\sqrt{3} U I \cos (\varphi) \Rightarrow P=\sqrt{3} U I 0.85 \Rightarrow P=1.47 U I
$$

The values of current intensity and voltage were recorded by means of the inverter software.

According to the proposed relationship (see Eq. (1)), a plot from calculated data is presented in coordinates $\left(P, f\right.$ and $\left.P / V_{L}, f\right)$ in Fig. 2.

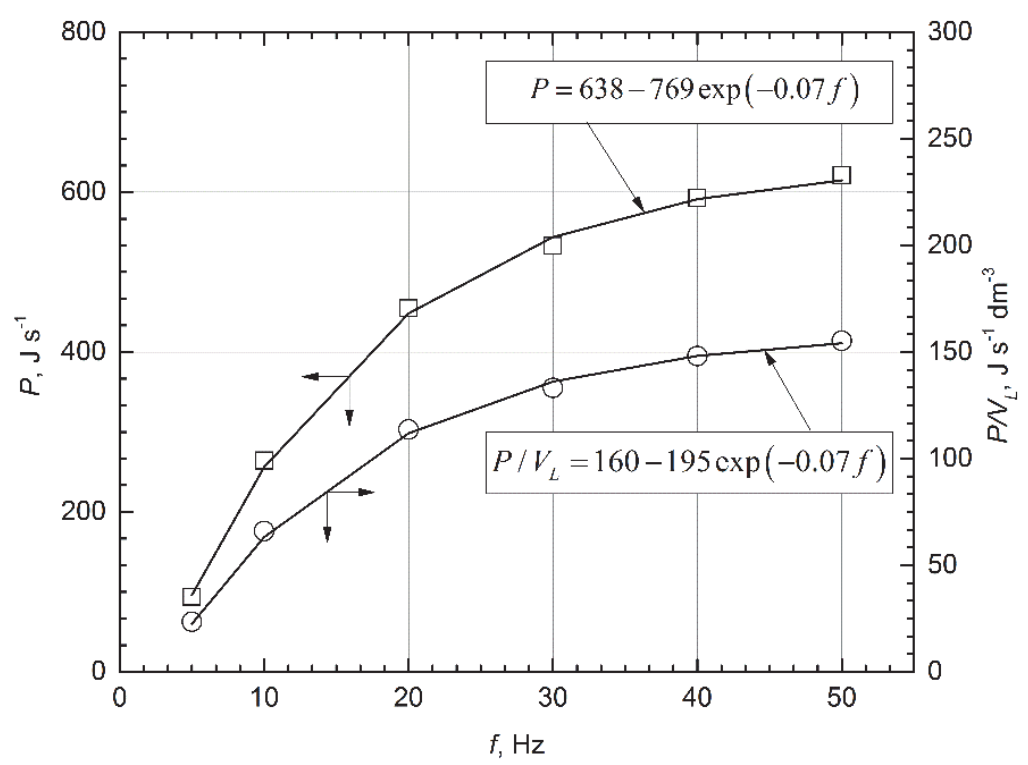

Fig. 2. The graphical presentation of relations between the calculated active power and the specific power input versus the RMF frequency

In the case of the current study, the RMF was identified by means of the magnetic induction $B$. The values of magnetic induction $B$ at different points inside the vessel were measured using the Hall probe connected and controlled by the frequency of the ac excitation current, $f$. A typical example of the magnetic field distribution in the cross-section of the RMF generator is given in Fig. 3a. Based on experimental measurements, the relationships of the maximum and the averaged values of the magnetic induction versus the frequency of the excitation current are shown in Fig. $3 b$.

The volumetric mass transfer coefficient, $k_{L} a$, was determined using the well-known dynamic gassingin method (Chistii, 1989). Two independent measurements were made simultaneously by means of two 
oxygen probes (localization is given in Fig. 1). The coefficient $k_{L} a$ was calculated as the averaged value from these measurements. The fluid was deoxygenated with nitrogen until the dissolved oxygen concentration had declined below $2 \%$ of air saturation. At that point the nitrogen flow was stopped, the present flow of air established and the dissolved oxygen concentration measured until the liquid became saturated with oxygen to the initial level.

a)

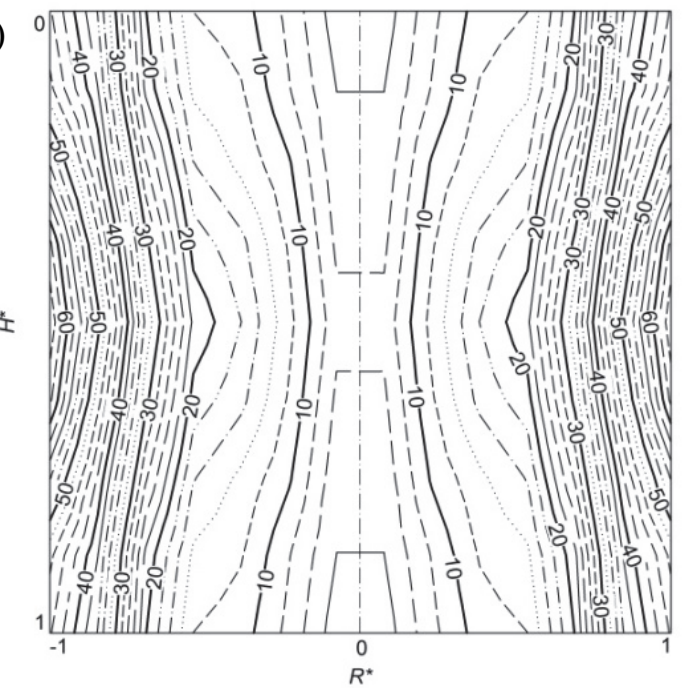

b)

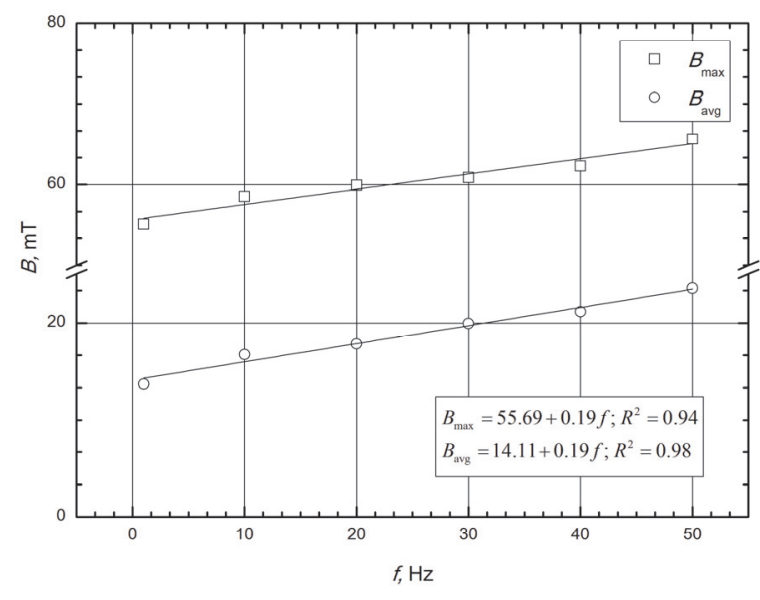

Fig. 3. A typical example of spatial distribution of the magnetic field in the cross-section of the RMF generator for $f=30 \mathrm{~Hz}$ (a) and the graphical presentation of the relations between the maximal $\left(B_{\max }\right)$ and the averaged $\left(B_{\text {avg }}\right)$ values of magnetic induction versus the frequency of the excitation current $f(\mathrm{~b})$

The volumetric mass transfer coefficient, $k_{L} a$, was determined on the basis of the following expression (Heim et al., 1995):

$$
\frac{d c}{d t}=k_{L} a\left(c^{*}-c\right)
$$

Taking into consideration that the oxygen transfer coefficient was constant throughout the process, the above Eq. (2) was integrated to:

$$
k_{L} a=-\frac{1}{t-t_{0}} \ln \left(\frac{c^{*}-c}{c^{*}-c_{0}}\right)
$$

\section{RESULTS AND DISCUSSION}

The proposed correlations for the volumetric mass transfer coefficient in mechanically mixers are usually presented in the following form (Galaction et al., 2004b):

$$
k_{L} a=f\left(\frac{P}{V_{L}}, w_{s}\right) \Rightarrow k_{L} a=p_{1}\left(\frac{P}{V_{L}}\right)^{p_{2}}\left(w_{s}\right)^{p_{3}}
$$

In the case of these experimental results, the total power input $\mathrm{P}$ was equal to the active power PRMF as defined by Eq. (1) (Rakoczy, 2013).

In order to provide a comparison of the mass transfer performance of the tested liquids, the experimental volumetric mass transfer coefficients $k_{L} a$ are plotted in Fig. 4. 

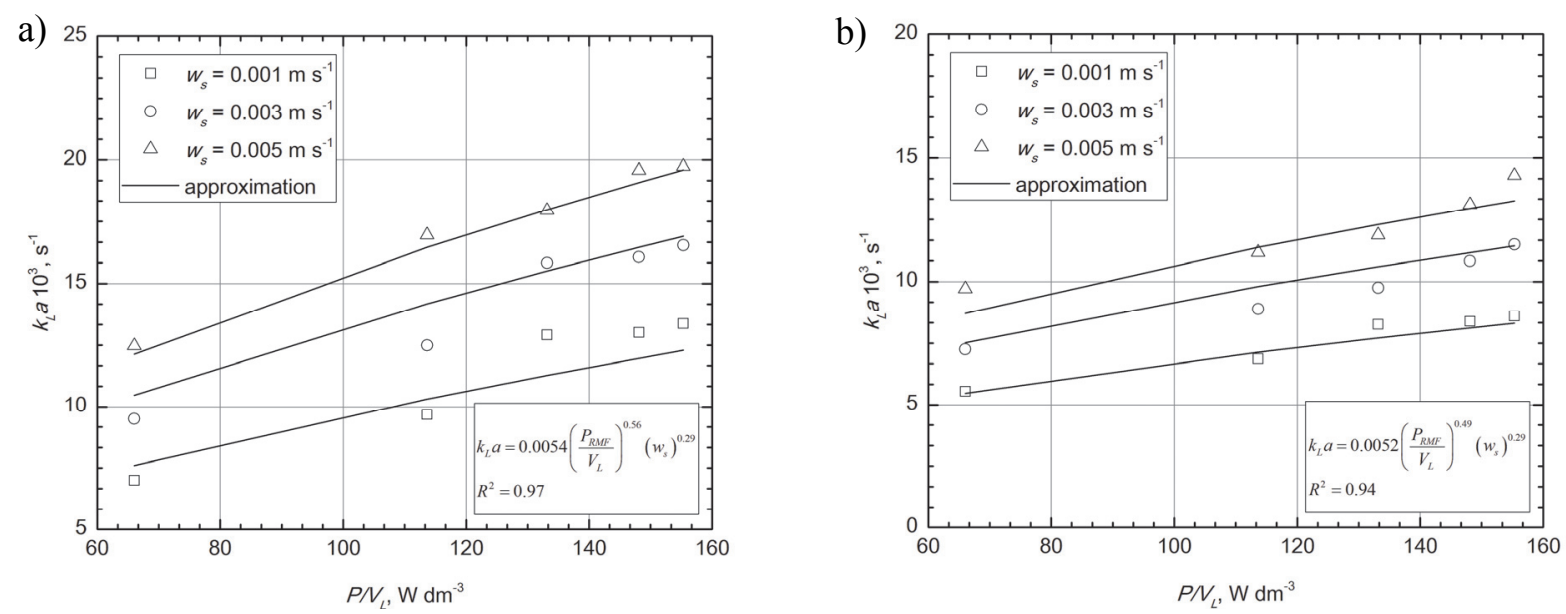

Fig. 4. The volumetric mass transfer coefficient versus the specific power input for distilled water (a) and HS medium (b)

Figure 4 shows that for a particular value of $w_{s}$, at equivalent $\mathrm{P} / \mathrm{V}_{\mathrm{L}}$, both liquids were characterized by different values of $k_{L} a$. Figure 4 also shows that the increase of specific power input increased the volumetric mass transfer coefficient for the distilled water (Fig. 4a) and the H-S medium (Fig. 4b). This parameter was enhanced by increasing aeration and specific power. The dependence of the oxygen mass transfer rate on the specific power is more pronounced for the distilled water. As shown in Fig. 4, the increase of the specific power led to the significant intensification of oxygen mass transfer.

The obtained results are compared with available experimental data for the standard mixer (Karimi et al., 2013). Fig. 5 demonstrates that the mixer which produces higher values of $k_{L} a$ is the Rushton type turbine (single and dual impellers) for the tested aeration rates. The mass transfer data obtained for the mixer with a dual Rushton turbine is consequently higher than the data obtained for a single impeller and magnetically assisted aeration process.

In the present work, the gas-liquid mass transfer under the action of RMF may be correlated by the following relationship (Li et al., 2006)

$$
k_{L} a=f\left(B_{\text {avg }}, w_{s}\right)
$$

Based on the experimental results, it can be assumed that the volumetric mass transfer coefficient is the function of magnetic induction and the superficial gas velocity.

a)

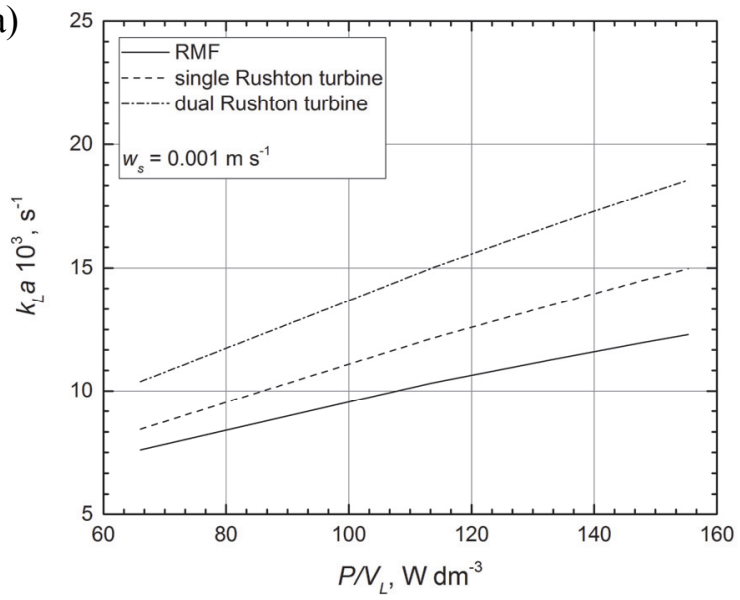

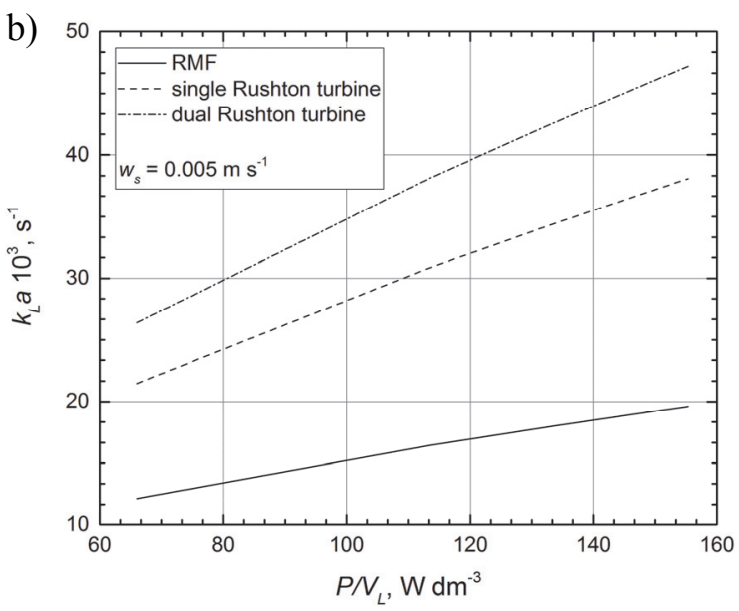

Fig. 5. The performance comparison of the obtained results for distilled water (with RMF application) with literature data from viewpoint of volumetric mass transfer coefficient (two types of impellers - single and dual Rusthon turbines) 
The following correlation was developed to estimate $k_{L} a$ for the distilled water and the HS medium, respectively:

$$
\begin{gathered}
\left.k_{L} a\right|_{\text {water }}=0.0035\left(B_{\text {avg }}\right)^{1.11}\left(w_{s}\right)^{0.32} \\
\left.k_{L} a\right|_{H-\text { Smedium }}=0.0028\left(B_{\text {avg }}\right)^{1.03}\left(w_{s}\right)^{0.31}
\end{gathered}
$$

The $k_{L} a$ measurement data are presented as a function of the averaged value of the magnetic induction and the superficial gas velocity in Fig. 6. As can been seen, the volumetric mass transfer coefficient did not react very sensitively on the airflow modulation. In contrast it more sensitively responded to changes in magnetic induction. Fig. 6 also shows that over a wide interval of the averaged magnetic induction, the application of distilled water as the working liquid led to significantly higher $k_{L} a$ values than the HS medium at the same magnetic induction.

The influence of the magnetic field on the volumetric mass transfer coefficient may be described by means of the amplification factor (Galaction et al, 2004b). The effect observed due to the magnetic field can be described using the ratio between the volumetric mass transfer coefficient under the RMF action, $\left(k_{L} a\right)_{B a v g=v a r}$, and for the volumetric mass transfer coefficient without the RMF action, $\left(k_{L} a\right)_{B a v g=0}$, obtained for similar experimental conditions.
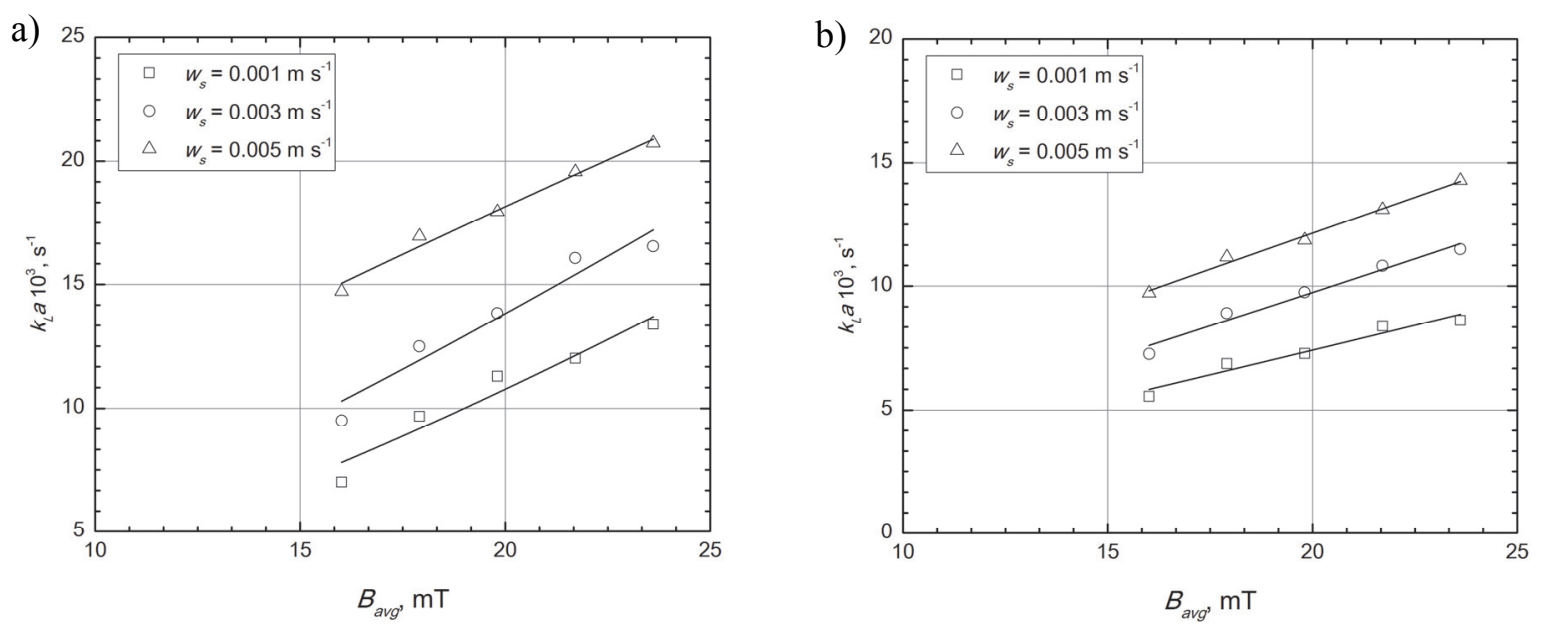

Fig. 6. The volumetric mass transfer coefficient versus the averaged magnetic induction for distilled water (a) and HS medium (b)

The variation in the coefficient $k_{L} a$ depending on the volumetric flow rate of air is presented in Fig. 7.

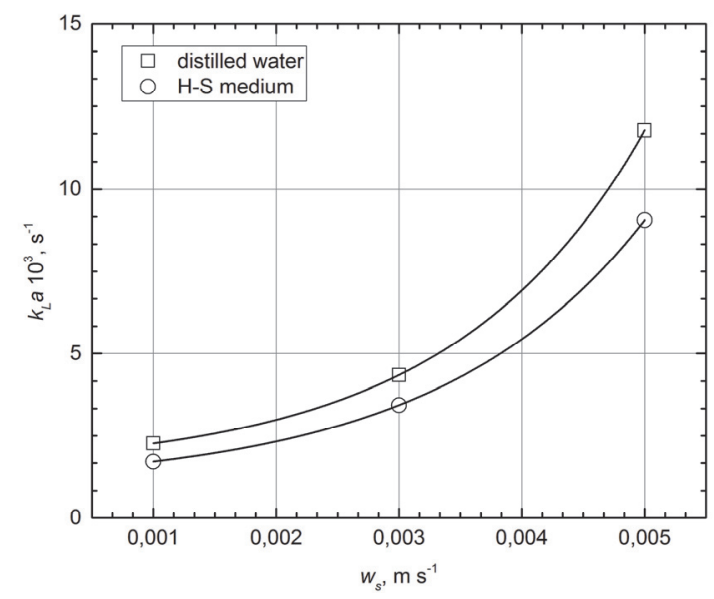

Fig. 7. The volumetric mass transfer coefficient versus the superficial gas velocity 
a)

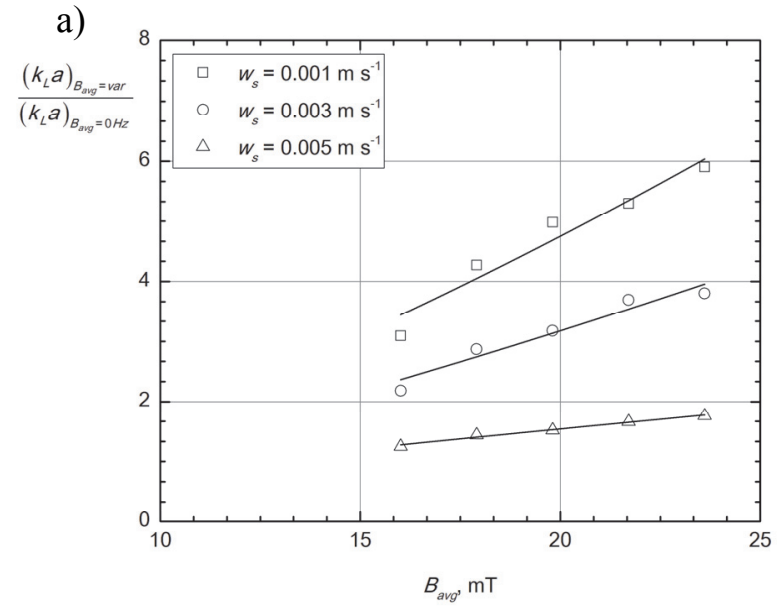

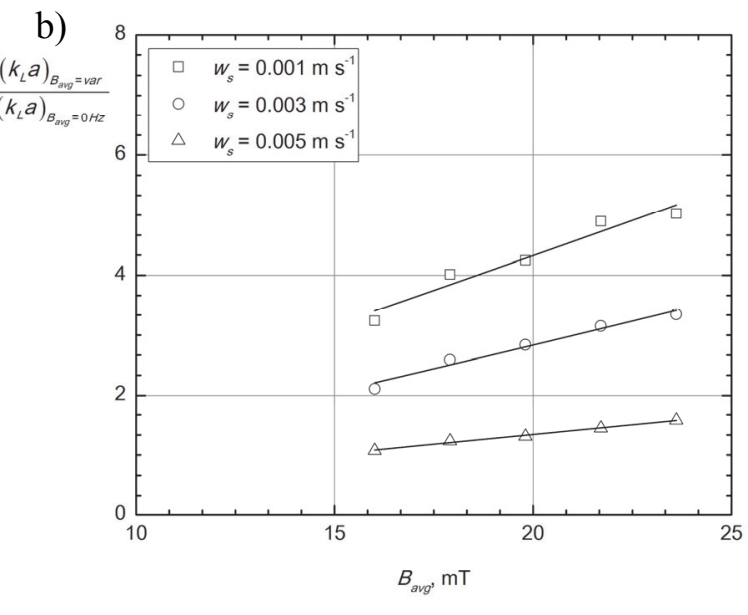

Fig. 8. The effect of the averaged magnetic induction on the amplification factor of the volumetric mass transfer coefficient

Figure 8 shows the ratio $\left(k_{L} a\right)_{\text {Bavg }=v a r} /\left(k_{L} a\right)_{\text {Bavg }=0}$ obtained with aeration and in the presence of RMF. Figure 8 also shows that RMF caused a significant enhancement of the volumetric mass transfer coefficient as compared to the $k_{L} a$ value for the working liquids without the RMF action.

For a better characterization of the RMF generator according to the oxygen mass transfer, the term of oxygen transfer efficiency, $E_{\mathrm{O} 2}$, may by applied and defined as (Chisti et al., 2002)

$$
E_{O_{2}}=\frac{k_{L} a}{\left(\frac{P}{V_{L}}\right)}
$$

As can be seen form Figures 9a and 9b, plotted for the two considered liquids, the variation of the oxygen transfer efficiency with the specific power input was contrary to that of $k_{L} a$ with the specific power input (see Fig. 4). These results suggest that the oxygen mass transfer rate reaches higher values under the action of RMF, but with considerable energy consumption. As shown in Fig. 9 for the airdistilled water and the air-H-S medium systems, the increase of the aeration rate is connected with the increase of the oxygen transfer efficiency due to the enhancement of turbulence and of the extent of the interfacial area needed for oxygen transfer.

a)

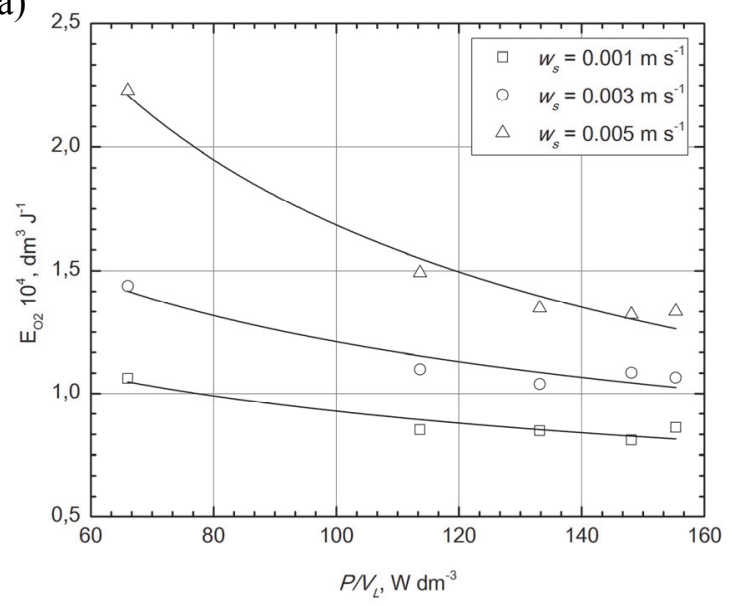

b)

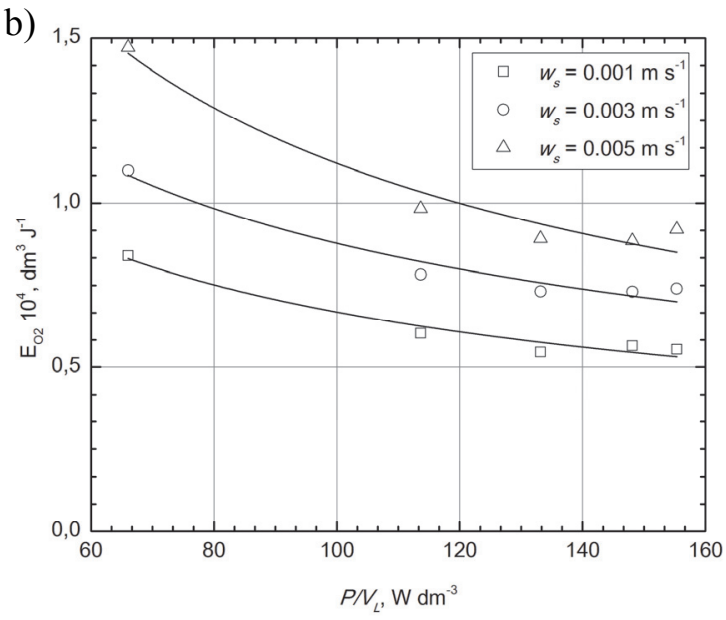

Fig. 9. The influence of specific power input on oxygen transfer efficiency

The obtained results may be explained by the fact that apart from molecular diffusion, fluid vorticity is the only mixing mechanisms of the liquid bulk in gas-liquid contractor (Hajiani et al., 2013a). It should 
be noticed that the electrically conductive fluid exposed to alternating MF (including RMF) induce the Lorentz electromagnetic force. This force which is generated perpendicularly to the electric field, acts as the driving force inducing currents inside the liquid and causing liquid rotation (Moffat, 1965; Rakoczy, 2011; Spitzer, 1999; Volz et al., 1999).

The movement of the medium exposed to RMF can be explained on the basis of micro-level dynamo concept (Gaafar, 2008). The RMF associated eddy currents can be induced in the liquid as a consequence of the magnetic field because the liquid contains various charged particles e.g. ions (Anton-Leberre et al., 2010; Hristov 2010). Eddy currents may generate local MFs around ions, which in combination with an externally-applied MF, cause induction of their rotation and thus the movement of the liquid in accordance to the MF vector. As a consequence of this process, rotating ions create "dynamos" which cause the effect of micro-mixing (Anton-Leberre et al., 2010; Mehedintu et al. 1997). This mixing effect of RMF can enhance the mass transfer by reducing the diffusion mass transfer resistance (Hristov, 2010). Hristov (2010) indicated that the hydrodynamic effects on the mass transfer in magnetically assisted bioreactors are of primary importance. It was also suggested that the coefficient $k_{L} a$ can be effectively controlled by the magnetic field. Iwasaka et al. (2004) showed that the magnetic field gradient in the horizontal direction causes a convectional flow, which increases oxygen concentration in the liquid. It was suggested that the magnetic field can be applied for the motion control of diamagnetic and paramagnetic elements in the liquid phase (Iwasaka et al., 2004). Kitazawa et al. (2001) clearly indicated that the application of magnetic field significantly enhances oxygen concentration in water dissolved from the gas phase. It has turned out that dissolution kinetics can be enhanced several times by the magnetic field. A possible explanation for the enhancement of $k_{L} a$ factor under the RMF action might be that the increase of $k_{L} a$ is mainly due to the possibility of keeping bubbles small in size (Al-Qodah et al., 2000). Weng et al. (1992) reported that bubble rise velocity and bubble diameter decrease with the magnetic field strength. It was suggested that the magnetic field retains bubbles in liquid and it might increase the surface tension of bubbles, thereby enhancing the lifetime of bubbles (Fabian, 2002).

\title{
4. CONCLUSIONS
}

Results obtained in the present study indicated that RMF provided higher values of the volumetric mass transfer coefficient $k_{L} a$ in the analyzed liquids (distilled water and HS medium). This parameter increased along with the magnetic field strength and the superficial gas velocity. The intensification of aeration led to the increase of turbulence inside the analyzed liquids, thus promoting the acceleration of oxygen transfer rate. Therefore, it can be concluded, that modifying the specific power input between $60-160 \mathrm{~W} \cdot \mathrm{dm}^{-3}$ and the superficial air velocity from 0.001 to $0.005 \mathrm{~m} \cdot \mathrm{s}^{-1}$, the maximum $k_{L} a$ can be increased by 6 times in the case of distilled water and by 5 times in the case of the HS medium.

This study was supported by the National Centre for Research and Development in Poland (Grant No. LIDER/011/221/L-5/13/NCBR/2014).

The authors would like to thanks firm STAGUM-EKO for co-operation and technical support in the area of the membrane sparger.

\section{SYMBOLS}

\author{
$B_{\text {avg }} \quad$ vector magnitude of magnetic field, $\mathrm{kg} \cdot \mathrm{A}^{-1} \cdot \mathrm{s}^{-2}$ \\ $c \quad$ instantaneous concentration of dissolved oxygen, $\mathrm{kmol} \cdot \mathrm{m}^{-3}$
}


$c_{0} \quad$ initial concentration of dissolved oxygen, $\mathrm{kmol} \cdot \mathrm{m}^{-3}$

$c^{*} \quad$ saturation concentration of dissolved oxygen, $\mathrm{kmol} \cdot \mathrm{m}^{-3}$

I current intensity, A

$k_{L} a \quad$ volumetric mass transfer coefficient, $\mathrm{s}^{-1}$

$P \quad$ total power input, $\mathrm{W}$

$\mathrm{Pa} \quad$ active power, $\mathrm{J} \cdot \mathrm{s}^{-1}$

$p_{1}, p_{2}$ and $p_{3}$ constants for a given combination of the fluid and the RMF generator

$t \quad$ time, $\mathrm{s} ; \mathrm{t}_{0}$ - initial time, $\mathrm{s}$

U voltage, $\mathrm{V}$

$V_{L} \quad$ volume of liquid, $\mathrm{m}^{3}$

$w_{s} \quad$ superficial gas velocity, $\mathrm{m} \cdot \mathrm{s}^{-1}$

Greek symbols

$\cos (\varphi) \quad$ phase shift angle between voltage and current

\section{REFERENCES}

Al-Qodah Z., Al-Hassan M., 2000. Phase holdup and gas-to-liquid mass transfer coefficient in magneto stabilized G-L-S airlift fermenter. Chem. Eng. J., 79, 41-52. DOI: 10.1016/S1385-8947(00)00142-X.

Anton-Leberre V., Haanappel E., Marsaud N., Trouilh L., Benbadis L., Boucherie H., 2010. Exposure to high static or pulsed magnetic fields does not affect cellular processes in the yeast Saccharomyces cerevisiae. Bioelectromagnetics, 31, 28-38. DOI: 10.1002/bem.20523.

Chen C.M., Leu L.P., 2001. Hydrodynamics and mass transfer in three-phase magnetic fluidized beds. Powder Technol., 117, 198-206. DOI: 10.1016/S0032-5910(00)00370-3.

Chisti M.Y., 1989. Airlift Bioreactors. Elsevier, New York, USA.

Chisti M.Y., Jauregui-Haza U.J., 2002. Oxygen transfer and mixing in mechanically agitated airlift bioreactors. Biochem. Eng. J., 10, 143-153. DOI: 10.1016/S1369-703X(01)00174-7.

Ciechańska D., Struszczyk H., Gruzińska K., 1998. Modification of Bacterial Cellulose. Fibres Text. East. Eur., 6, 61-65.

El-Gaddar A., Frénéa-Robin M., Voyer D., Aka H., Haddour N., Krähenbühl L., 2013. Assesment of 0.5 T static files exposure effect on yeast and HEK cells using electrorotation. Biophys. J. 104, 1805-1811. DOI: 0.1016/j.bpj.2013.01.063.

Fabian A.C., 2002. Bubbles, flows and fields. Science, 296, 1040-1041. DOI: 10.1126/science.1072074.

Fijałkowski K, Żywicka A., Drozd R., Niemczyk A., Junka A. F., Peitler D., Kordas M., Konopacki M., Szymczyk P., El Fray M., Rakoczy R., 2015. Modification of bacterial cellulose through exposure to the rotating magnetic field. Carbohyd. Polym., 133, 52-60. DOI: 10.1016/j.carbpol.2015.07.011.

Gaafar E.S.A., Hanafy M.S., Tohamy E.Y., Ibranhim M.H., 2008. The effect of electromagnetic field on protein molecular structure of E. Coli and its pathogenesis. Rom. J. Biophys., 18, 145-169.

Galaction A.-I., Dan C., Comelin O., Marius T., 2004a. Enhancement of oxygen mass transfer in stirred bioreactors using oxygen-vectors: 1. Simulated fermentation broths. Bioproc. Biosyst. Eng., 26, 231-238. DOI: 10.1007/s00449-004-0353-5.

Galaction A.-I., Cascaval D., Oniscu C., Turnea M., 2004b. Prediction of oxygen mass transfer coefficients in stirred bioreactors for bacteria, yeasts and fungus broths. Biochem. Eng. J., 20, 85-94. DOI: 10.1016/j.bej.2004.02.005.

Garcia-Ochoa F., Gomez E., 2009. Bioreactor scale-up and oxygen transfer rate in microbial processes: An overview. Biotechnol Adv., 27, 153-176. DOI: 10.1016/j.biotechadv.2008.10.006.

Gorobets S.V., Yu G.O., Demianeneko I.V., Nikolaenko R.N., 2013. Self-organization of magnetic nanoparticles in providing Saccharomyces cerevisiae yeasts with magnetic properties. J. Magn. Magn. Mater., 337-338, 5357. DOI: $10.1016 /$ j.jmmm.2013.01.004.

Hajiani P., Larachi F., 2013a. Remotely excited magnetic nanoparticles and gas-liquid mass transfer in Taylor flow regime. Chem. Eng. Sci., 93, 257-265. DOI:10.1016/j.ces.2013.01.052. 
Hajiani P., Larachi F., 2013b. Giant effective liquid-self diffusion in stagnant liquids by magnetic nanomixing. Chem. Eng. Process., 71, 77-82. DOI: 10.1016/j.cep.2013.01.014.

Heim, A., Kraslawski, A., Rzyski, E., Stelmach, J., 1995. Aeration of bioreactors by self-aspirating impellers. Chem. Eng. J. Bioch. Eng., 58, 59-63. DOI:10.1016/0923-0467(94)06093-2.

Hristov J., 2010. Magnetic field assisted fluidization - A unified approach. Part 8. Mass Transfer: Magnetically assisted bioprocesses. Rev. Chem. Eng., 26, 55-128. DOI: 10.1515/REVCE.2010.006.

Hristov J., Perez V.H., 2011. Critical analysis of data concerning Saccharomyces cerevisiae free-cell proliferations and fermentations assisted by magnetic and electromagnetic fields. Int. Rev. Chem. Eng., 3, 3-20.

Iwasaka M., Ikchata M., Miyakoskhi, Ueno S., 2004. Strong static magnetic field effects on yeast proliferation and distribution. Bioelectrochemistry, 65, 59-68. DOI: 10.1016/j.bioelechem.2004.04.002.

Karimi A., Golbabaei F., Mehrnia M. R., Neghab M., Mohhamad K., Nikpey A., Pourmand M. R., 2013. Oxygen mass transfer in a stirred tank bioreactor using different impeller configurations for environmental purposes. Iran. J. Environ. Healt., 10, 6. DOI: 10.1186/1735-2746-10-6.

Kitazawa K., Ikezoe Y., Uetake H., Hirota N., 2001. Magnetic field effects on water, air and powder. Physica B, 294-295, 709-714. DOI: 10.1016/S0921-4526(00)00749-3.

Li W., Zong B. N., Li X. F., Meng X. K., Zhang J. L., 2006. Interphase mass transfer in G-L-S magnetically stabilized bed with amorphous alloy SRNA-4 catalyst. Chinese J. Chem. Eng., 14, 734-739. DOI: 10.1016/S1004-9541(07)60004-4.

Mehedintu M., Berg H., 1997. Proliferation response of yeast Saccharomyces cerevisiae on electromagnetic field parameters. Bioelectroch. Bioener., 43, 67-70. DOI: 10.1016/S0302-4598(96)05184-7.

Mills P.L., Chaudhari R.V., 1999. Reaction engineering of emerging oxidation processes. Catal. Today., 48, 17-29. DOI: $10.1016 / \mathrm{S} 0920-5861(98) 00354-\mathrm{X}$.

Moffat H.K., 1965. On fluid flow induced by a rotating magnetic field. J. Fluid. Mech., 22, 521-528. DOI: 10.1017/S0022112065000940.

Montes F.Y., Catalan J., Galan M.A., 1999. Prediction of $k_{L} a$ in yeast broths. Proc. Biochem., 34, 549-554. DOI: 10.1016/S0032-9592(98)00125-3.

Ozbek B., Gayik S., 2001. The studies on the oxygen mass transfer coefficient in a bioreactor. Proc. Biochem., 36, 729-741. DOI: 10.1016/S0032-9592(00)00272-7.

Rakoczy R., 2011. Theoretical and experimental analysis of the influence of the rotating magnetic field on the selected unit operations and processes of chemical engineering. Academic Publisher of West Pomeranian University of Technology, Szczecin. ISBN 978-83-7663-074-8.

Rakoczy R., 2013. Mixing energy investigations in a liquid vessel that is mixed by using a rotating magnetic field. Chem. Eng. Process. Process Intensif., 66, 1-11. DOI: 10.1016/j.cep.2013.01.012.

Rakoczy R., Konopacki M., Fijałkowski K., 2016. The influence of a ferrofluid in the presence of an external rotating magnetic field on the growth rate and cell metabolic activity of a wine yeast strain. Biochem. Eng. J. 109, 43-50. DOI: 10.1016/j.bej.2016.01.002.

Rakoczy, R., Masiuk, S., 2011. Studies of a mixing process induced by a transverse rotating magnetic field. Chem. Eng. Sci., 66, 2298-2308. DOI: 10.1016/j.ces.2011.02.021.

Santos L.O., Alegre R.M., Garcia-Diego C., Cuellar J., 2010. Effects of magnetic fields on biomass and glutathione production by the yeast Saccharomyces cerevisiae. Process. Biochem., 45, 1362-1367. DOI: 10.1016/j.procbio.2010.05.008.

Spitzer K.H., 1999. Application of rotating magnetic fields in Czochralski crystal growth. Prog. Cryst. Growth. Ch., 38, 59-71. DOI: 10.1016/S0960-8974(99)00008-X.

Torab-Mostaedi M., Safdari S.J., Moosavian M.A., Maragheh M.G., 2008. Mass transfer coefficients in a Hanson mixer-settler extraction column. Braz. J. Chem. Eng., 25, 473-481. DOI: 10.1590/S0104-66322008000300005.

Volz M. P., Mazuruk K., 1999. Thermoconvective instability in a rotating magnetic field. Int. J. Heat Mass Tran., 42, 1037-1045. DOI: 10.1016/S0017-9310(98)00168-9.

Weng D.C., Cheng L.N., Han Y., Zhu W.X., Xu S.M., Ouyang F., 1992. Continuous ethanol fermentation in a three-phase magnetic fluidized bed bioreactor. AIChE Symposium Series, Fluidized Processes: Theory and Practice, 88, 107-115. 